\title{
Numerical Simulation of Weakly Ionized Hypersonic Flow for Reentry Configurations
}

\author{
Leonardo C. Scalabrin* and Iain D. Boyd ${ }^{\dagger}$ \\ Department of Aerospace Engineering, University of Michigan, Ann Arbor, MI, 48109, USA
}

\begin{abstract}
Numerical simulations of axisymmetric flows over reentry configurations at hypersonic conditions using a Navier-Stokes solver are presented. The Navier-Stokes equations are modified using Park's two-temperature model to account for thermochemical nonequilibrium and weak ionization effects. The finite-volume method is used to solve the set of differential equations. The code has the capability to handle any mixture of hexahedra, tetrahedra, prisms and pyramids in $3 \mathrm{D}$ or triangles and quadrilaterals in $2 \mathrm{D}$. The results in this paper only use quadrilaterals. Numerical fluxes between the cells are discretized using a modified Steger-Warming Flux Vector Splitting approach which has low dissipation and is appropriate to calculate boundary layers. A point or line implicit method is used to perform the time integration. Pressure, heat transfer rates and electron number density profiles are compared to available experimental and flight measurements.
\end{abstract}

\section{Introduction}

$\mathrm{D}_{\mathrm{s}}^{\mathrm{s}}$ ETERMINING the extreme conditions experienced during reentry is an important part of a spacecraft design process. During reentry, the maximum temperature in the flowfield can reach values as high as $50,000 \mathrm{~K}$, leading to dissociation and ionization of gases. The degree of dissociation and ionization has important effects on the heat transfer to the spacecraft, the key element of thermal protection systems design. The amount of ionization is also important for the design of communication systems that reduce the amount of communication blackout during reentry. To a lesser degree, the dissociation and ionization of the gases in the atmosphere also impact the aerodynamic properties of the spacecraft. Accurate prediction of the aerodynamic coefficients is important for stability analysis and can reduce the recovery footprint of the spacecraft.

The simulation of weakly ionized gases over reentry vehicles has been performed successfully for a large number of spacecraft, including RAM-C, ${ }^{1}$ FIRE,${ }^{2,3}$ the Apollo Command Module, ${ }^{4}$ the Huygens probe ${ }^{5}$ and the Mars Space Laboratory. ${ }^{6}$ Today, numerical simulation of reentry flows is an integral part in the design process of reentry vehicles. ${ }^{7}$ A design environment usually has a limited time to develop or test new models and new methods. In an academic environment, the time constraints are reduced facilitating the development of new models and methods for the physical processes that occur during reentry. The final test of any such model is its impact on design variables when integrated in a full numerical simulation of a reentry vehicle. Hence, it is desirable to develop a numerical code able to perform hypersonic reentry calculations in order to test new physical models.

This paper presents the advances obtained in developing a numerical code able to simulate hypersonic flows in thermochemical nonequilibrium that will serve as test bed for new physical models to be developed in the future. The current code is an extension of an earlier version of the code ${ }^{8}$ modified to handle weakly

${ }^{*}$ Graduate Student, Student Member AIAA.

${ }^{\dagger}$ Professor, Associate Fellow AIAA 
ionized gases. This paper presents some results obtained using the code to simulate weakly ionized flow over hypersonic reentry configurations.

\section{Modeling}

The flow is modeled assuming that the continuum approximation is valid and that the fluid can be weakly ionized. Furthermore, it is assumed that the rotational and translational energy modes of all species can be described by a single temperature $T$ and that the vibrational energy mode of all species and the electron energy can be described by a single temperature $T_{v}$. With those approximations, the conservation equations for the two-dimensional system can be written as ${ }^{1,9-11}$

$$
\frac{\partial Q}{\partial t}+\frac{\partial\left(F-F_{v}\right)}{\partial x}+\frac{\partial\left(G-G_{v}\right)}{\partial y}=S_{c v}
$$

where

$$
Q=\left\{\begin{array}{lllllll}
\rho_{1} & \cdots & \rho_{n s} & \rho u & \rho v & E & E_{v}
\end{array}\right\}^{T}
$$

is the vector of conserved variables. In these expressions, $\rho_{1} \cdots \rho_{n s}$ are the species densities, $u$ and $v$ are the bulk velocity components, $E$ is the total energy per unit volume of mixture given by

$$
E=\sum_{s \neq e} \rho_{s} C v_{s} T+\frac{1}{2} \rho\left(u^{2}+v^{2}\right)+\sum_{s \neq e} \rho_{s} h_{s}^{o}+E_{v} \quad,
$$

where $C v_{s}$ is the species specific heat at constant volume, subscript $e$ indicates electrons, $h_{s}^{o}$ is the species heat of formation at $0 \mathrm{~K}$ and

$$
E_{v}=\sum_{s \neq e} \rho_{s} e_{v s}+\rho_{e} e_{e}
$$

is the vibrational energy per unit volume. In the last equation, the species vibrational energy per unit mass, $e_{v s}$, is modeled using a harmonic oscillator for which

$$
e_{v s}=\frac{R u}{M_{s}} \frac{\theta_{v s}}{\exp \left(\theta_{v s} / T_{v}\right)-1},
$$

where $R u$ is the universal gas constant, $M_{s}$ is the species molecular weight and $\theta_{v s}$ is the harmonic oscillator characteristic temperature. The electron energy, $e_{e}$, is given by

$$
e_{e}=C v_{e} T_{v}
$$

The electronic energy of atoms and molecules is neglected in the present work due to the relatively small temperatures achieved in the flows considered in this paper.

The inviscid and viscous flux vector components in the $x$ direction are given by

$$
F=\left\{\begin{array}{c}
\rho_{1} u \\
\vdots \\
\rho_{n s} u \\
\rho u^{2}+p \\
\rho u v \\
(E+p) u \\
E_{v} u
\end{array}\right\} \quad \text { and } \quad F_{v}=\left\{\begin{array}{c}
-\rho_{1} u_{d 1} \\
\vdots \\
-\rho_{n s} u_{d n s} \\
\tau_{x x} \\
\tau_{x y} \\
\tau_{x x} u+\tau_{x y} v-\left(q_{t x}+q_{r x}+q_{v x}\right)-\sum\left(\rho_{s} h_{s} u_{d s}\right) \\
-q_{v x}-\sum\left(\rho_{s} e_{v s} u_{d s}\right)
\end{array}\right\},
$$


where $p$ is the pressure, $\tau_{i j}$ are the viscous stress components, $q_{t i}, q_{r i}$ and $q_{v i}$ are the translational, rotational and vibrational heat fluxes in the $i$-th direction. Moreover, $h_{s}$ is the species enthalpy and $u_{d s}$ is the species diffusion velocity in the $x$-direction. The flux vectors in the other directions are similar.

The pressure is calculated assuming that each species can be modeled using a perfect gas relation and Dalton's law of partial pressures so that

$$
p=\sum_{s \neq e} \rho_{s} \frac{R u}{M_{s}} T+\rho_{e} \frac{R u}{M_{e}} T_{v}
$$

The species enthalpies are given by

$$
h_{s}= \begin{cases}\left(C v_{t s}+C v_{r s}+R_{s}\right) T+h_{s}^{o}+e_{v s} & \text { for molecules } \\ \left(C v_{t s}+R_{s}\right) T+h_{s}^{o} & \text { for atoms } \\ \left(C v_{t e}+R_{e}\right) T_{v} & \text { for electrons }\end{cases}
$$

where $C v_{t s}$ and $C v_{r s}$ are the translational and rotational species specific heats at constant volume.

The viscous stresses are modeled assuming a Newtonian fluid for which, using Stokes' hypothesis,

$$
\tau_{i j}=\mu\left(\frac{\partial u_{j}}{\partial x_{i}}+\frac{\partial u_{i}}{\partial x_{j}}\right)+\lambda \nabla \vec{u} \delta_{i j} \quad, \quad \lambda=-\frac{2}{3} \mu \quad,
$$

where $\mu$ is the mixture coefficient of viscosity. The heat fluxes are modeled according to Fourier's law as

$$
\vec{q}_{t, r, v}=-\kappa_{t, r, v} \nabla T_{\mathrm{t}, \mathrm{r}, \mathrm{v}},
$$

where $\kappa_{t, r, v}$ are the mixture thermal conductivity for each energy mode. The species mass diffusion fluxes are modeled using Fick's law as

$$
\rho_{s} \vec{u}_{d s}=-\rho D_{s} \nabla Y_{s} \quad,
$$

where $D_{s}$ is the species diffusion coefficients and $Y_{s}$ is the species mass fraction.

Two different models to calculate mixture transport properties are available. One uses Wilke's semiempirical mixing rule ${ }^{12}$ with species viscosities calculated using Blottner's model ${ }^{13}$ and species thermal conductivities determined using Eucken's relation. ${ }^{14}$ The other option uses Gupta's mixing rule ${ }^{15}$ with species viscosities and thermal conductivities calculated using non-coulombic/coulombic collision cross section data compiled by Wright. ${ }^{16}$ The diffusion coefficients for all species are assumed equal as a simple way to ensure that the sum of diffusion fluxes is equal to zero. ${ }^{17}$ The diffusion coefficient is calculated assuming that the Lewis number, $L e$, is constant. In this work, it is assumed that $L e=1.4$.

The source term, $S_{c v}$, is given by

$$
S_{c v}=\left\{\begin{array}{lllllll}
\dot{w}_{1} & \cdots & \dot{w}_{n s} & 0 & 0 & 0 & \dot{w}_{v}
\end{array}\right\}^{T}
$$

where $\dot{w}_{1} \cdots \dot{w}_{n s}$ are the species mass production rates by chemical reactions and $\dot{w}_{v}$ is the vibrational energy source term. The species mass production rates are modeled using a standard finite-rate chemistry model for reacting air in conjunction with Park's two-temperature model ${ }^{11}$ to account for thermal nonequilibrium effects on the reaction rates. The forward reaction rates used in this work are taken from Ref. 18 or 19 and the backward reaction rates are calculated using curve fits for equilibrium constants taken from Ref. 11 or 19.

The vibrational energy source term is given by

$$
\dot{w}_{v}=-p_{e} \nabla \cdot \vec{u}+\sum \dot{w}_{s} e_{v s}+S_{t-v}+S_{h-e},
$$

where $p_{e} \nabla \cdot \vec{u}$ is an approximation to the work done on electrons by the electric field induced by the electron pressure gradient ${ }^{1,10}$ and $\dot{w}_{s} e_{v s}$ is the non-preferential addition or removal of vibrational energy of molecules 
by recombination or dissociation respectively. The energy exchange rate between the translation-rotational and the vibrational energy modes, $S_{t-v}$, is modeled using the Landau-Teller model ${ }^{14}$ and Millikan and White's correlation ${ }^{20}$ with exceptions for some pairs of interactions. ${ }^{18}$ A high temperature correction for limiting equivalent collision cross sections is also employed. ${ }^{21}$ The energy transfer rate between heavy particles and electrons, $S_{h-e}$, is given by

$$
S_{h-e}=3 R u \rho_{e}\left(T-T_{v}\right) \sqrt{\frac{8 R u T_{v}}{\pi M_{e}}} \sum_{r \neq e} \frac{\rho_{r} N_{a}}{M_{r}^{2}} \sigma_{e r},
$$

where $\sigma_{e r}$ are the collision cross sections between electrons and heavy particles. ${ }^{1,9}$

The finite-volume method applied to unstructured grids is used to solve the set of differential equations. ${ }^{22}$ The present code is capable of simulating 2D/Axisymmetric flows using any mixture of quadrilaterals and triangles, and 3D flows using any mixture of hexahedra, tetrahedra, prisms and pyramids. For axisymmetric simulations, the source terms that arise from the finite-volume integration of the system of equations are taken from Ref. 23. The inviscid fluxes across cell faces are discretized using a modified form of the StegerWarming Flux Vector Splitting (FVS) scheme ${ }^{24}$ which is less dissipative and yields better results in boundary layers. The method switches to the original Steger-Warming FVS scheme ${ }^{25,26}$ at shock waves by using a pressure switch. ${ }^{27}$ The viscous terms are calculated using the values of properties at the cell centers and at the nodes. ${ }^{28}$ The property values at the nodes are calculated using a simple average of the values of the cells that share that node. Use of this method increases the stencil employed in the derivative calculations in order to avoid loss of accuracy when using unstructured meshes. ${ }^{28}$ Time integration is performed using a point implicit or a line implicit method. ${ }^{29-31}$

\section{Higher order extension of inviscid fluxes}

Higher-order of accuracy extensions of finite volume codes using Flux Vector Splitting (FVS) or Riemann solvers to discretize the inviscid fluxes is straightforward in structured grids because of the implicit connectivity information. The MUSCL variable extrapolation ${ }^{22}$ originally developed for $1-\mathrm{D}$ calculations is readily extrapolated for 2 dimensions when using a structured grid. For the faces aligned to the vertical direction in the structured grid shown in Fig. 1, the values to the left $\left(u_{L}\right)$ and to the right $\left(u_{R}\right)$ are obtained according to

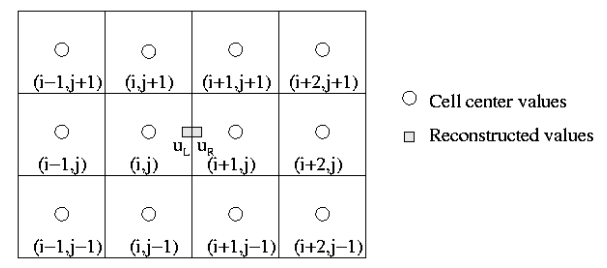

Figure 1. 2nd order stencil for a structured grid.

$$
\left\{\begin{array}{l}
u_{L}=u_{(i, j)}+\frac{1}{2} \Psi\left(r_{L}\right)\left(u_{(i, j)}-u_{(i-1, j)}\right) \\
u_{R}=u_{(i+1, j)}-\frac{1}{2} \Psi\left(r_{R}\right)\left(u_{(i+2, j)}-u_{(i+1, j)}\right)
\end{array},\right.
$$

where

$$
r_{L}=\frac{u_{(i+1, j)}-u_{(i, j)}}{u_{(i, j)}-u_{(i-1, j)}} \quad, \quad r_{R}=\frac{u_{(i+1, j)}-u_{(i, j)}}{u_{(i+2, j)}-u_{(i+1, j)}}
$$

and $\psi(r)$ is a limiter function. ${ }^{22}$ Even though all the meshes used in this work are "structured like" grids, the code handles them internally as unstructured grids. Hence, the implicit connectivity information - the 
information of each cell neighbors - characteristic of structured codes is not available making the above extension to higher order of accuracy more complicated.

Many approaches are available in the literature to achieve high order of accuracy using unstructured grids. The most well known and widely used is the one developed by Barth and Jespersen. ${ }^{32}$ Many authors modified the ideas of Barth and Jespersen to create their own extension to high order of accuracy. ${ }^{33,34}$ Those approaches are highly effective at transonic and low supersonic flows but they seem to fail at hypersonic flows due to the presence of stronger shocks. In fact, it is a common strategy in hypersonic flow simulations to use some sort of pressure sensor to totally turn off the higher order extension across strong shock waves. ${ }^{35,36}$ Though effective, the use of a pressure sensor should not be necessary because the main function of a limiter used in MUSCL extrapolation is to turn off the higher order terms close to shock waves.

A different approach to higher order extension using unstructured grids was developed by Batina ${ }^{37}$ in which a structured stencil is assembled locally so Eq. 16 can be used. One example of such a stencil is shown in Fig. 2(a). In that figure, the points $i-1$ and $i+2$ are the opposite vertices of the triangles that share the face for which the fluxes are being computed. The values at the vertices are obtained by averaging the cell center values of the cells that share the vertices. A similar idea is also used by Bibb et al ${ }^{38}$ in the FELISA cell-vertex code. Though FELISA is a $3 \mathrm{D}$ code, the idea is exemplified for a $2 \mathrm{D}$ case in Fig. 2(b). The coordinates of point $i-1$ in that figure are obtained by a reflection of the vector $i, i+1$ in relation to the point $i$. The property values at the point $i-1$ are obtained from interpolation of the values at vertices that define the cell in which the point lays. A problem with these two approaches is that the values of variables in some points of the localized stencil are obtained by interpolation or averaging. Close to shock waves, the interpolated or averaged quantities can be too large hindering the limiting process.

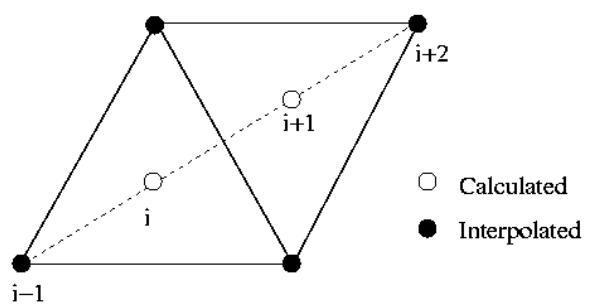

(a) Batina's

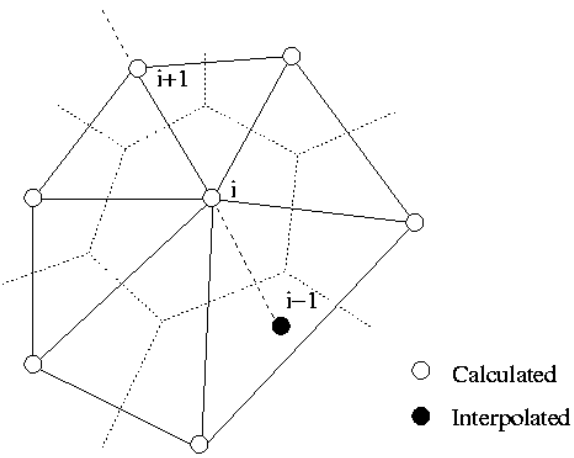

(b) Bibb et al

Figure 2. Stencil search approach for 2nd order calculations.

The approach proposed here follows the idea of Batina ${ }^{37}$ and Bibb et $\mathrm{al}^{38}$ closely. However, the interpolation step is eliminated. Instead, the stencil is constructed such that only cell centered values are used. The search for the stencil is depicted in Fig. 3 for "structured like" and unstructured grids. This figure shows the search for the cell $i+2$. The search for the cell $i+2$ is limited to cells that share at least one node with cell $i+1$. The cells that meet such restriction are shaded in Fig. 3. The selected cell will be the one such that the dot product between the face normal and the normalized vector joining the face centroid to the cell centroid is the maximum positive value. Figure 3 shows some of the face centroid-cell centroid vectors and the selected cells. For the search of the cell $i-1$, the dot product must be the maximum negative value.

It should be noted that the distance between the cells $i+1$ and $i+2$ centroids is different from the distance between $i$ and $i+1$. That is clearly the case in Fig. 3(b). Therefore, the undivided differences used 
to calculate the limiter must be corrected according to

$$
r=\frac{u_{i+1}-u_{i}}{u_{i+2}-u_{i+1}} \frac{b}{a}
$$

where $\|a\|=\left\|\vec{r}_{i+1}-\vec{r}_{i}\right\|$ and $\|b\|=\left\|\vec{r}_{i+2}-\vec{r}_{i+1}\right\|$. It is important to note that the search for the stencil is performed only once at the start of the code run. This is in contrast to $\mathrm{ENO}^{39,40} / \mathrm{WENO}^{41}$ schemes that search for stencils at every iteration. The cost for the stencil search is negligible in relation to the total run.

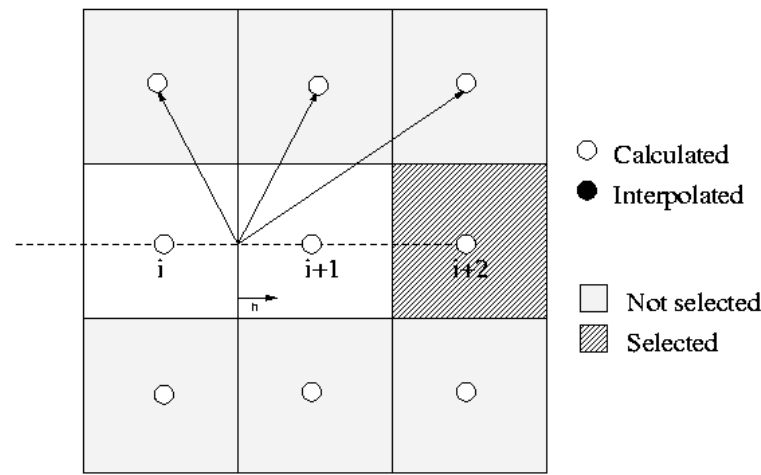

(a) Quadrilaterals

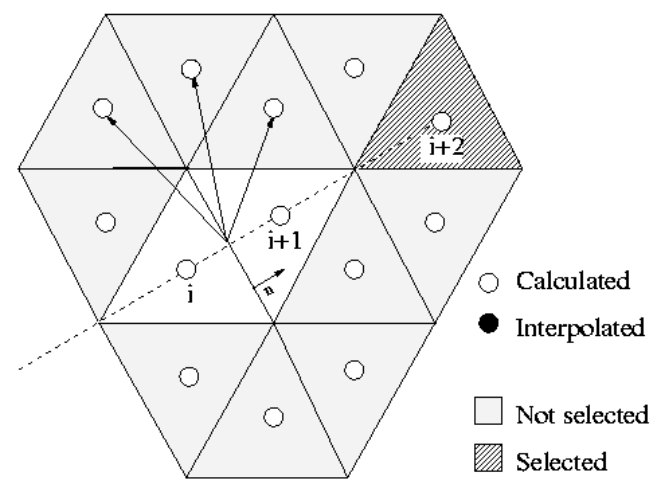

(b) Triangles

Figure 3. Second-order stencil search.

\section{Implicit Line Solver}

The system of equations used to numerically simulate hypersonic, chemically reacting viscous flows is very stiff. ${ }^{22}$ One of the options to overcome the time-step limitations in such a case is using implicit time integration. The developed code can use a point-implicit or a line-implicit method to solve the set of equations implicitly. The line implicit method has better convergence performance but it comes at the expenses of a more complicated algorithm.

A line in the system of equations to be solved can be written as

$$
A_{i} \Delta Q_{i}+\sum_{j} B_{j} \Delta Q_{n b}=R_{i}^{n} \quad,
$$

where $A$ and $B$ contain the inviscid and viscous jacobians, $R$ is the residual of the system of equations and $\Delta Q$ is the correction used in the time march. The summation in the last expression is over all $j$-th faces that form the $i$-th cell. Moreover, $n b$ stands for the neighbor of the $i$-th cell that shares the $j$-th face. Details of the formulation can be obtained in Ref. 8 or 23. When using unstructured grids, Eq. 19 is a sparse matrix without any structure. Considering the mesh shown in Fig. 4(a), the corresponding matrix form of Eq. 19 is shown in Fig. 5. There are many algorithms available for efficient solution of sparse linear systems. Such algorithms rely heavily on general mathematical properties of matrices to solve the system. These algorithms, however, do not take into account some flow characteristics that can simplify the system. For instance, flow gradients will be stronger in the direction normal to the body according to boundary layer theory. It was shown ${ }^{29}$ that solving the system of equations exactly in the normal direction for structured grids and relaxing the system in the other directions was a good option to parallelize an implicit code.

$$
6 \text { of } 18
$$


The idea of solving the linear system originated by an implicit method using special directions in unstructured grids is not new. ${ }^{31,36}$ The first step in such a method is to define the special directions that will be used. In this work, such directions are called lines that are grown from the body surface by a simple algorithm that chooses the next cell such that the line stays nearly normal to the body. Cells that are "leftover" are grouped together and handled by a point implicit algorithm. The lines formed from the mesh in Fig. 4(a) are indicated in Fig. 4(b). That figure also shows a renumbering of the cells that will be shown to facilitate the solution of the linear system. The renumbering of the cells is never carried out in the code. Instead, an array keeps track of the relation between the two cell numbering systems.

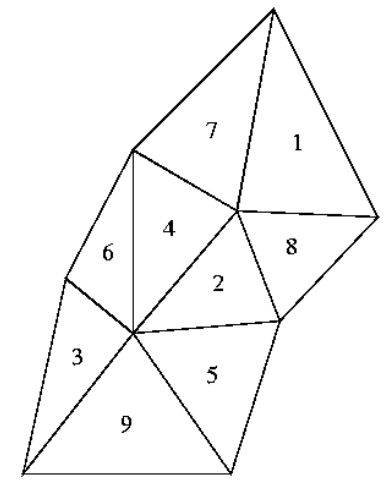

(a) Mesh

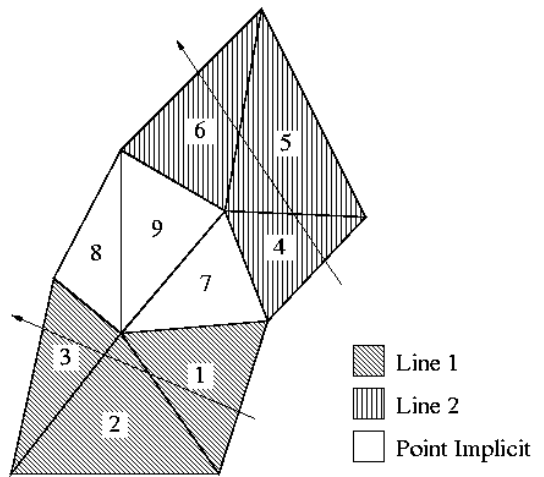

(b) Linear system

Figure 4. Distinct numbering used in the code.

Using the numbering for the cells provided in Fig. 4(b), the sparse system is greatly simplified as can be observed in Fig. 6. The matrix is still sparse, but one can see a tridiagonal structure embedded in the matrix. In order to take advantage of the tridiagonal structure, an iterative process is assembled. All the terms off the tridiagonal part of the matrix are moved to the right hand side of the equation. By doing that, the iterative process is now of the form depicted in Fig. 7. The last three lines of the system represent the three cells "leftover" from the line finding procedure. Those cells corrections are calculated using a simple point implicit method which is naturally integrated in the tridiagonal solver. Due to its diagonal characteristic,

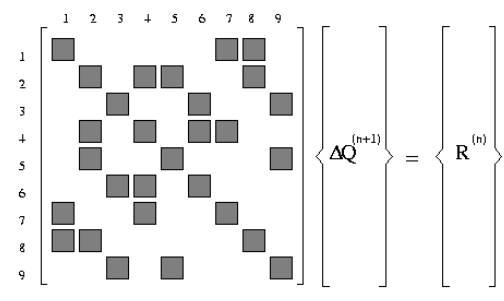

Figure 5. Typical sparse linear system for unstructured grids.

the system is very simple to solve. Assuming that $\Delta Q^{(n+1,0)}=0, \Delta Q^{(n+1,1)}$ is obtained by solving a simple tridiagonal system of equations. The next step requires a multiplication of the sparse matrix on the right hand side by the newly calculated $\Delta Q^{(n+1,1)}$. That multiplication is straightforward when using the face based data structure of the mesh and the mesh numbering. The result of that multiplication is mapped to the linear system numbering and a new right hand side is fed to the tridiagonal solver. Following Ref. 29, 


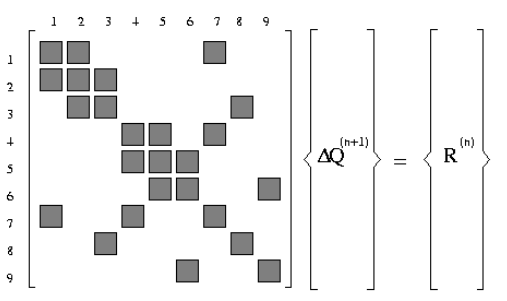

Figure 6. Full linear system.

four iterations are taken in the iterative process. The code starts running with a very small $C F L$ number which is increased to a maximum value of 10 when using a second order scheme or 1000 when using a first order scheme.

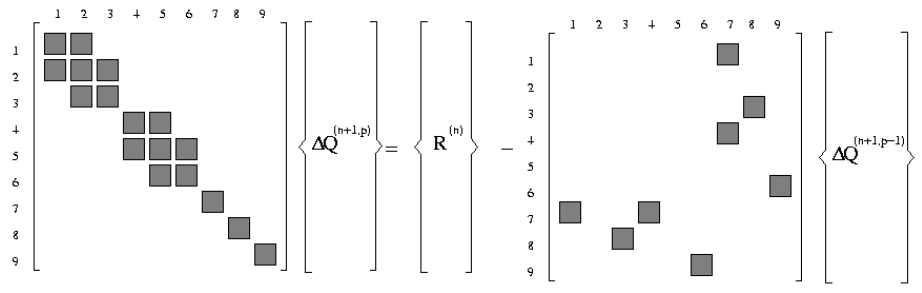

Figure 7. Iterative solution of linear system.

\section{Parallel Processing}

The code developed in this work takes full advantage of today's parallel machines by dividing the computational work among many processors. This task is accomplished using METIS ${ }^{42}$ to partition the mesh between the processors and MPI to communicate necessary information from one processor to another.

METIS uses graph theory to partition unstructured grids minimizing the amount of inter-processor communication. Each node in the graph corresponds to a cell in the mesh. Each edge in the graph connecting two nodes corresponds to the face shared by the cells represented by the two nodes. A partition generated this way usually is not optimal for a line implicit solver because most of the lines are significantly shortened. In order to avoid such a problem, the graph is modified so that each node in fact corresponds to a line and each edge corresponds to all the faces connecting one line to another. The weights of the nodes in the graph are set to the number of cells that form the line and the weight of each edge is equal to the number of faces connecting each line so that the load balancing of the partitions is not reduced.

The implemented algorithm is highly effective as can be observed in Fig. 8 which shows the partitions for a hybrid unstructured grid and for a "structured like" grid. It is clear in these figures that lines growing normal to the body can reach their maximum length before reaching an inter-processor boundary.

\section{Results}

To demonstrate the physical modeling capabilities of the code, three sets of data are used for comparisons. The first set consists of heat transfer values over an experimental model of a Mars entry spacecraft ${ }^{43-45}$ measured in the HYPULSE Expansion Tube. The second set comprises in-flight measurements of electron number densities over a reentry spacecraft. ${ }^{1}$ The third set consists of experimental measurements of heat

\section{8 of 18}

American Institute of Aeronautics and Astronautics 


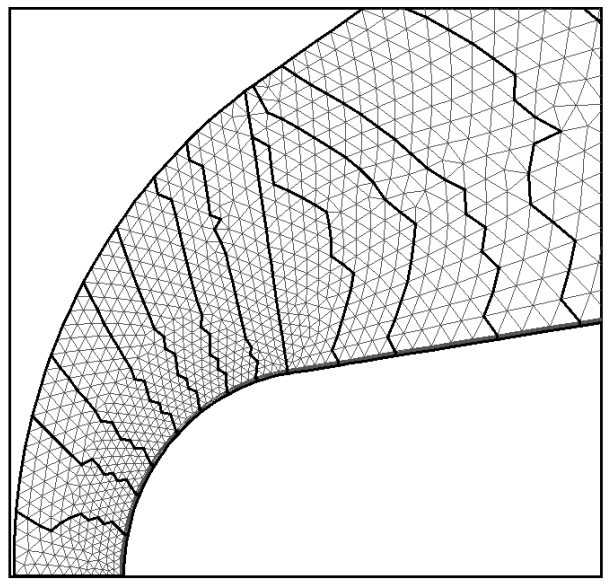

(a) Unstructured

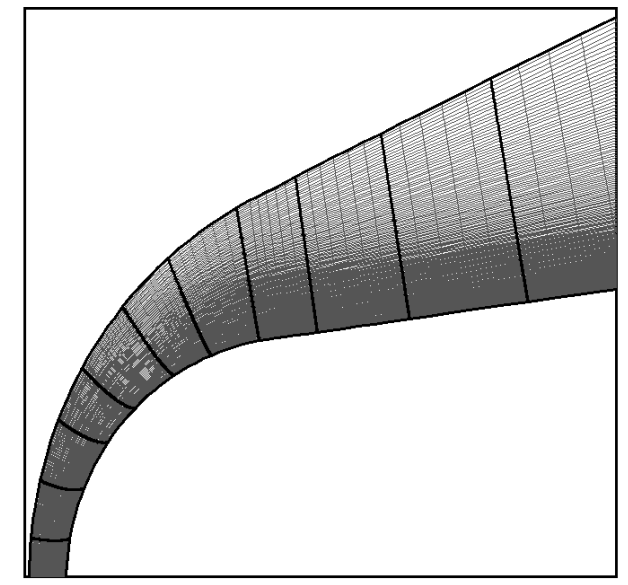

(b) Structured

Figure 8. Mesh partitioning.

transfer, pressure and electron number densities over a blunted cone ${ }^{46,47}$ taken in the CALSPAN 96" shock tunnel. The freestream conditions for all the mentioned data sets are tabulated in Table 1. The table also includes frozen freestream conditions calculated for the same conditions of the CALSPAN data set. ${ }^{48}$ All conditions satisfy the limit for a continuum approximation - $K n<0.01$ and all of them are in the laminar regime.

\section{A. HYPULSE Mars experimental model}

This excellent set of data ${ }^{45}$ was successfully used in code comparisons ${ }^{44}$ previously. The data set has heat transfer values for the forebody and afterbody flow over a Mars entry vehicle experimental model. Afterbody flow results are very sensitive on the mesh used. ${ }^{44}$ Because this work does not include mesh adaptation procedures, only the forebody flow is simulated. Details about the model geometry can be found in Ref. 43 . The mesh used in this simulation has 200 points in the normal direction and 102 points along the geometry. This test case is simulated using a 5 species air model with 17 reactions. ${ }^{11}$ Figure 9(a) shows the axial velocity contours over the geometry forebody which highlight the bow shock wave in front of the body. In addition, it can be observed that the shock standoff distance is very small - in this case around $2 \mathrm{~mm}-\mathrm{a}$ characteristic of hypersonic flows. The low velocity region extends until the flow starts to expand at the geometry shoulder. An earlier attempt in simulating this test case ${ }^{8}$ using a 1st order scheme did not yield good results. The results of that simulation are shown in Fig. 9(b) which also includes experimental values and the solution generated by the present code. The improvement in the results is remarkable. The heat transfer values calculated using the 2 nd order scheme proposed in this work falls within the experimental uncertainties for most of the distribution. 
Table 1. Freestream Conditions.

\begin{tabular}{rrrrr}
\hline & HYPULSE & RAM-C II & \multicolumn{2}{c}{ CALSPAN } \\
\hline & & & Equilibirum & Frozen \\
\hline$Y_{N_{2}}$ & $7.670 \times 10^{-1}$ & $7.670 \times 10^{-1}$ & $7.670 \times 10^{-1}$ & $7.348 \times 10^{-1}$ \\
$Y_{O_{2}}$ & $2.330 \times 10^{-1}$ & $2.330 \times 10^{-1}$ & $2.330 \times 10^{-1}$ & $1.867 \times 10^{-1}$ \\
$Y_{N O}$ & 0.0 & 0.0 & 0.0 & $6.482 \times 10^{-2}$ \\
$Y_{N}$ & 0.0 & 0.0 & 0.0 & $9.918 \times 10^{-5}$ \\
$Y_{O}$ & 0.0 & 0.0 & 0.0 & $1.360 \times 10^{-2}$ \\
$Y_{N O^{+}}$ & 0.0 & 0.0 & 0.0 & $7.313 \times 10^{-9}$ \\
$Y_{e^{-}}$ & 0.0 & 0.0 & 0.0 & $1.337 \times 10^{-13}$ \\
$T_{\infty}(K)$ & 1113.0 & 254.0 & 510.1 & 510.1 \\
$\rho_{\infty}\left(k g / m^{3}\right)$ & $5.710 \times 10^{-4}$ & $2.70236 \times 10^{-4}$ & $2.911 \times 10^{-3}$ & $2.911 \times 10^{-3}$ \\
$u_{\infty}(m / s)$ & 5162.0 & 7650.0 & 4229 & 4229 \\
$T_{w}(K)$ & 300.0 & 1200.0 & 300.0 & 300.0 \\
$M_{\infty}$ & 7.9 & 23.9 & 9.32 & 9.27 \\
$R e_{d}$ & $3.39 \times 10^{4}$ & $1.95 \times 10^{4}$ & $1.38 \times 10^{5}$ & $1.38 \times 10^{5}$ \\
$K n$ & $2.8 \times 10^{-4}$ & $1.2 \times 10^{-3}$ & $2.9 \times 10^{-4}$ & $2.9 \times 10^{-4}$ \\
\hline
\end{tabular}

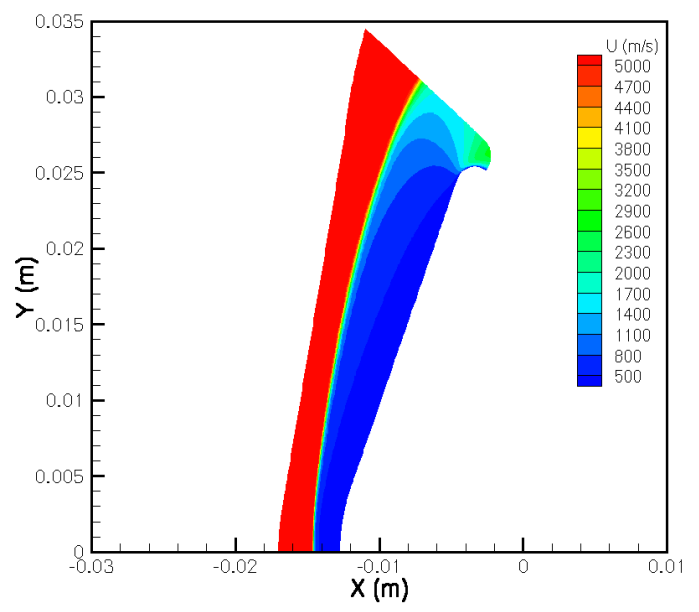

(a) Axial velocity contours

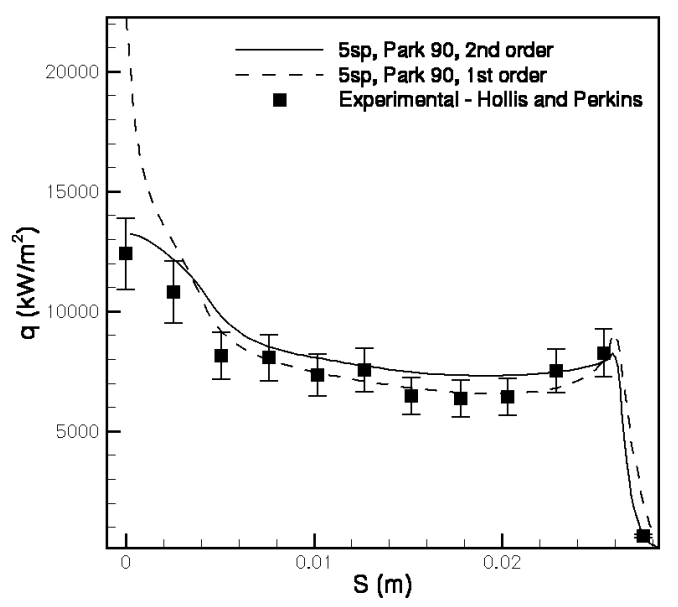

(b) Heat transfer comparisons

Figure 9. Numerical results for HYPULSE Mars experimental model. 


\section{B. RAM-C II flight data}

The RAM-C II flight data is another good source of measurements for code comparisons. This set of data has been used extensively for code assessment. ${ }^{1,49,50}$ In this work, the data obtained at $61 \mathrm{~km}$ altitude is used in the comparisons. Geometry details can be obtained in Ref. 1. The mesh used in this work has 120 points in the normal direction and 60 points along the vehicle surface. Two different air models are used in this study. ${ }^{11,19}$ Figure 10 shows a brief overview of the results obtained in the numerical simulation of this set. In particular, Fig. 10(a) highlights the influence of the high temperature correction (HTC) of Millikan and White's vibrational relaxation time. One can observe that without the use of the correction, the energy transfer between the translational-rotational and the vibrational mode is so fast that the vibrational temperature reaches temperatures almost as high as the translational temperature. The high vibrational temperature leads to stronger dissociation in relation to the case using the high temperature correction which causes a smaller shock standoff distance, also observed in Fig. 10(a).

Maximum electron number densities in the normal direction are compared to flight data and other numerical simulations in Fig. 10(b). The electron number density is very high in the stagnation region and falls rapidly as the flow temperature reduces due to the expansion around the spheric nose cap. The present numerical results are in good agreement with the numerical results of Grasso and Capano ${ }^{49}$ which used a 2 temperature model as well. That work used forward reaction rates from Ref. 11 and equilibrium constants from Ref. 19. The present results using the two different chemistry models bound the distribution from Grasso and Capano. The numerical results of Candler ${ }^{1}$ and Josyula and Bailey ${ }^{50}$ use a 6 temperature model - one temperature for each molecule vibrational energy and another temperature for the electron energy. Not surprisingly, their calculations yield different results from the present simulation. Moreover, their work use the ambipolar assumption to model the diffusion coefficients of the ionized gas. Considering the uncertainty in the data given by the electrostatic probe measurements, all the numerical calculations seem to be within the flight data error. The results by Josyula and Bailey are in excellent agreement with the experimental data.

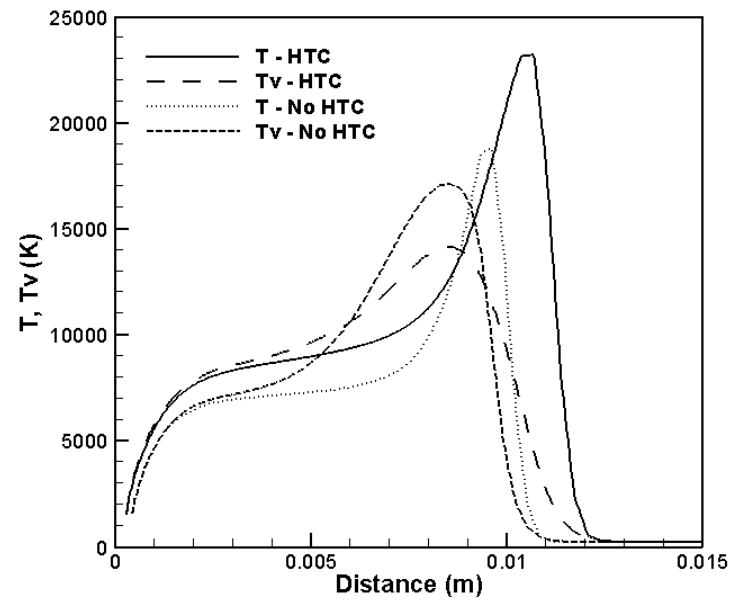

(a) Temperatures along stagnation line

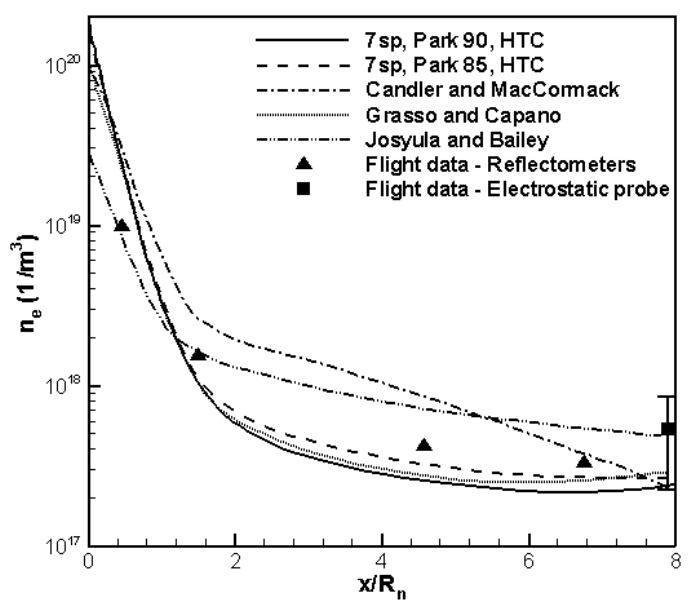

(b) Electron number density comparisons

Figure 10. Numerical results for RAM-C II flight conditions at $61 \mathrm{~km}$ altitude. 


\section{CALSPAN blunted-cone experiments}

Geometry information for the blunted-cone simulated is available in Ref. 46. Two different meshes are used in this study. Both meshes have 300 points in the normal direction and 90 points along the body. The heat transfer in the stagnation region is very sensitive to the grid. Close to the symmetry axis, the points along the body are distributed at every 3 degrees to avoid problems. ${ }^{51}$ Even though mesh "a" is fairly regular, it still caused problems in the computed heat transfer distribution. Mesh "a" is further modified so that all the cells at the wall have a constant height. The new mesh is called mesh "b" and can be seen in Fig. 11(b).

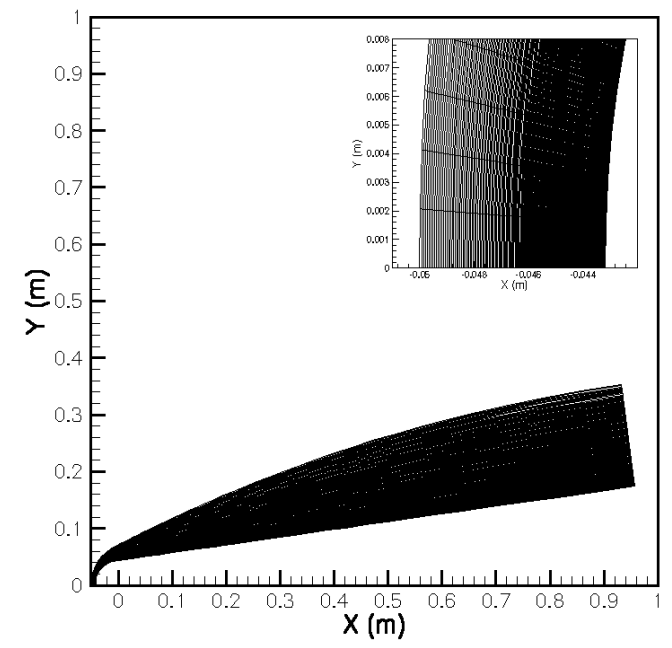

(a) Mesh "a"

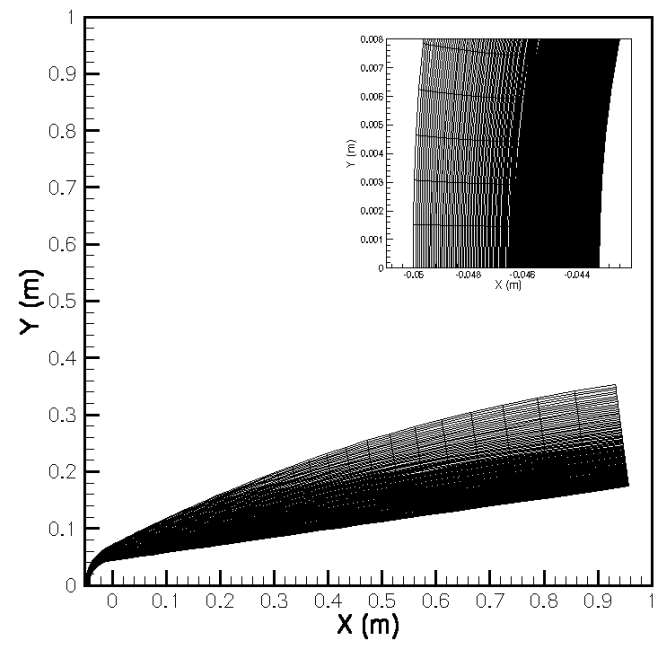

(b) Mesh "b"

Figure 11. Meshes used for the CALSPAN blunted cone calculations.

Figure 12(a) presents an overview of the flow over the blunted cone. A strong bow shock wave forms in front of the body. The small distance between the shock wave and the wall, around $5 \mathrm{~mm}$, comprises the shock layer characteristic of hypersonic flows. The flow rapidly expands around the spheric nose until it reaches the cone section. The strong shock wave causes a sudden increase in temperature, as depicted in Fig. $12(\mathrm{~b})$. The high temperature, around $8000 \mathrm{~K}$, causes dissociation and ionization reducing the temperature immediately after the shock wave, also seen in Fig. 12(b).

The numerical method used in the simulations has a significant impact on heat transfer values. Figure 13(a) shows the results for heat transfer along the body using 1st and 2nd order schemes, original and modified Steger-Warming FVS and different meshes. The heat transfer distribution calculated using the modified Steger-Warming FVS (MSW) with mesh "a" presents a sudden peak in the stagnation region. Using the original Steger-Warming FVS (OSW) reduces the peak but the heat transfer distribution is no longer flat close to the stagnation point. That is probably caused by the diffusive nature of the OSW FVS for low subsonic flows. The unphysical peak is eliminated when using MSW and mesh "b". The use of a first order scheme also yields an unphysical peak close to the stagnation region which is similar to what is observed for the HYPULSE simulation. The peak in the heat transfer obtained using mesh "a" is also eliminated by making the switch between the OSW and the MSW FVS more sensitive to pressure variations. The fact that both changing the mesh and the switch eliminates the peak suggests that the problem is related to the 


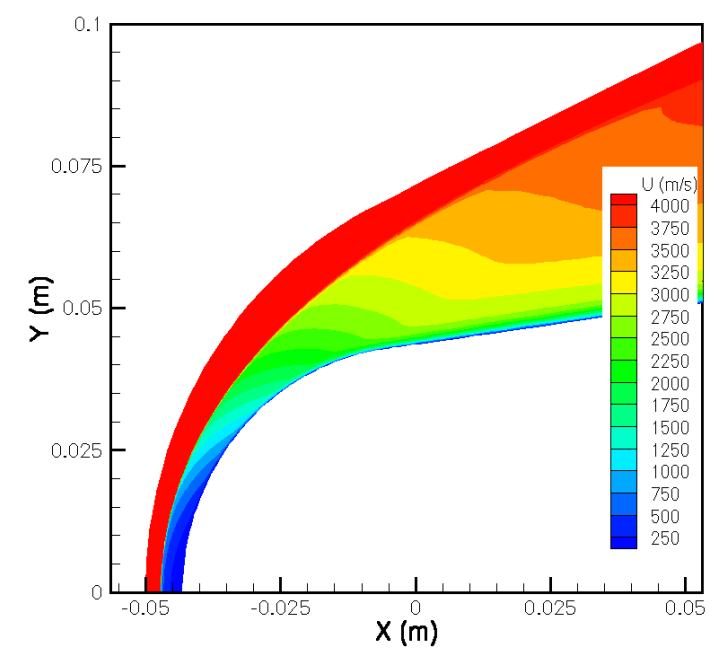

(a) Axial velocity

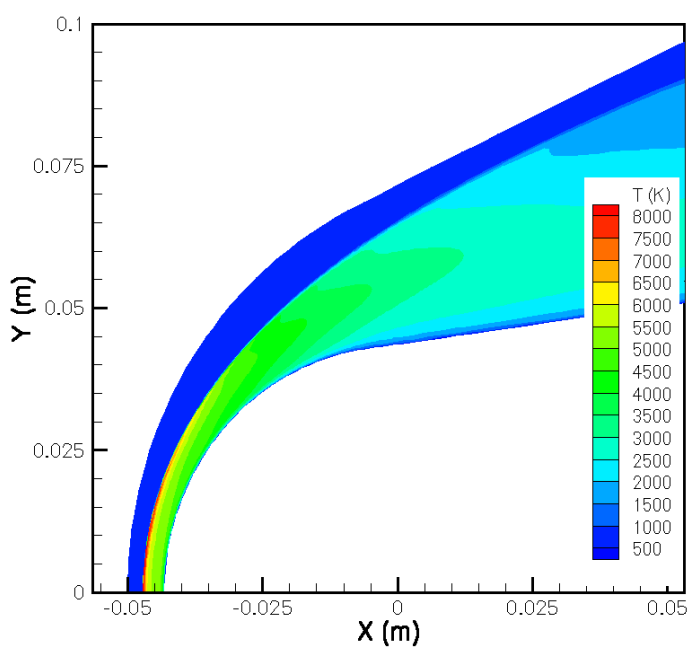

(b) Temperature

Figure 12. Flowfield over the CALSPAN blunted cone experimental model.

shock capturing scheme. Mesh "b" is such that the shock becomes aligned to the grid which requires less dissipation from the numerical algorithm. Increasing the switch sensitivity increases the dissipation in the numerical method such that the shock wave is successfully captured in mesh "a". The numerical methods do not have much impact on the pressure distribution along the body, as can be observed in Fig. 13(b).

The modeling choices also have a significant impact on the heat transfer distribution. Figure 14(a) shows that using Gupta's mixing rule yields larger values for heat transfer than using Wilke's mixing rule with Blottner's model because the latter underpredicts the transport properties. ${ }^{52}$ It can be observed also that modeling the freestream as frozen flow yields higher heat transfer results because the dissociation products originated in the high pressures and temperatures of the shock tunnel carry additional energy stored in chemical form. Due to the relatively lower temperatures, the use of a 11 species chemistry model does not influence the results significantly. Finally, assuming a supercatalytic wall, ${ }^{53}$ which assumes chemical equilibrium at the wall temperature, increases the heat transfer dramatically. It should be noted that a supercatalytic wall is an upper bound for the wall catalysis process. In reality, the catalysis process is limited by the amount of species diffusing to the wall. ${ }^{54}$ The experimental results for the heat transfer distribution are much larger than this upper limit which may indicate problems in the measurements. In fact, Ref. 47 acknowledges that attempts to simulate the same conditions did not generate satisfactory results. In contrast, the measured pressure distribution is in good agreement with the calculations, as seen in Fig. 14(b). This figure also indicates that the choices in physical modeling do not have any significant impact on the pressure distribution along the body.

The experiment by Chadwick provided electron number density profiles normal to the body at two different positions. The first position, ES-1, is at $0.198 \mathrm{~m}$ measured axially from the nose while the second position, ES-2, is at $0.47 \mathrm{~m}$ from the nose. The calculated and measured electron number density profiles for the ES-1 position are presented in Fig. 15(a). One can observe that all the calculated profiles under predict the measured values. The profiles calculated assuming that the freestream is in equilibrium conditions predict the smallest number densities. The profile calculated assuming a frozen freestream shows the shock 


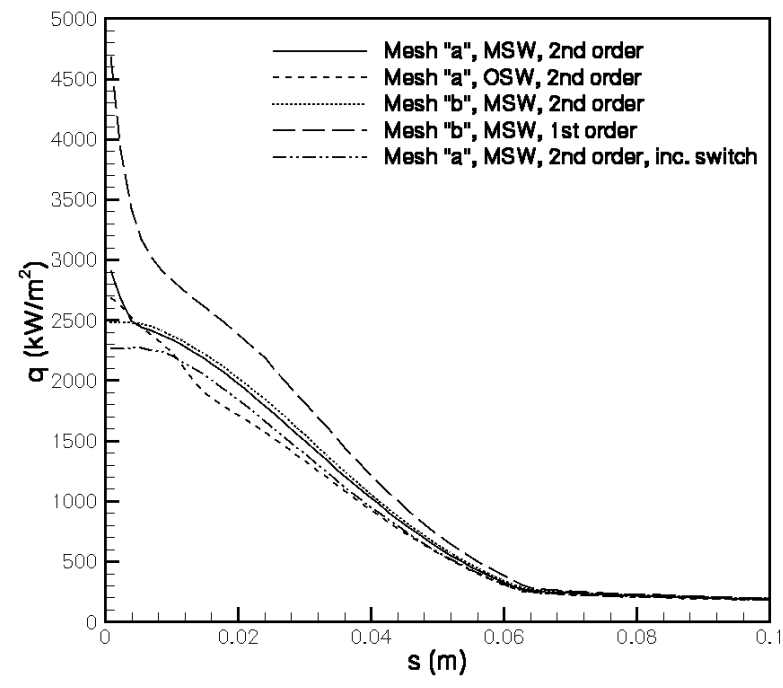

(a) Heat transfer

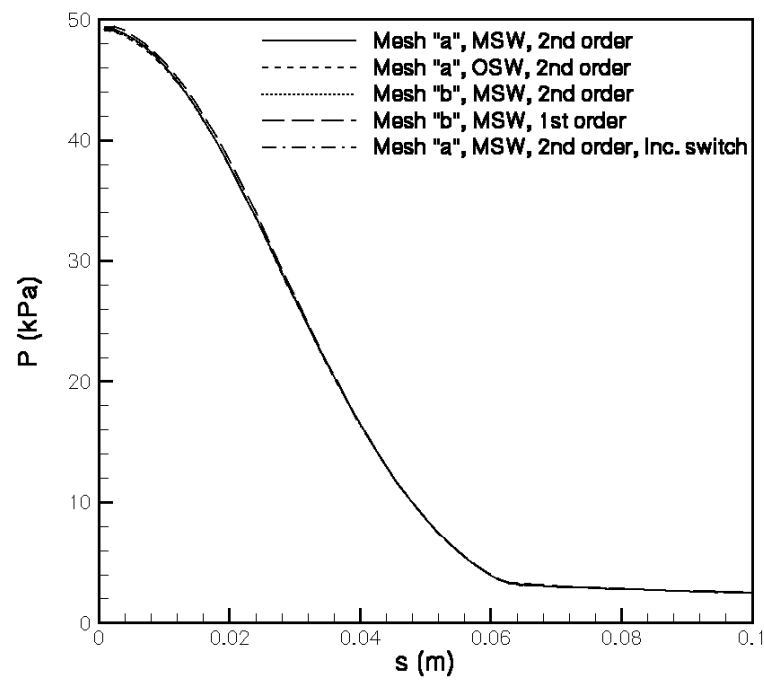

(b) Pressure

Figure 13. Dependence of surface properties on meshes and numerical methods.

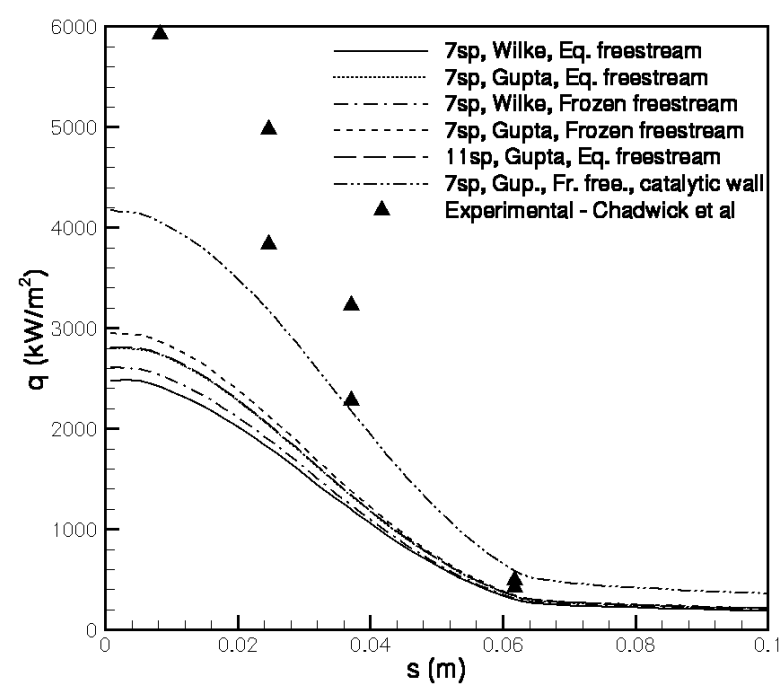

(a) Heat transfer

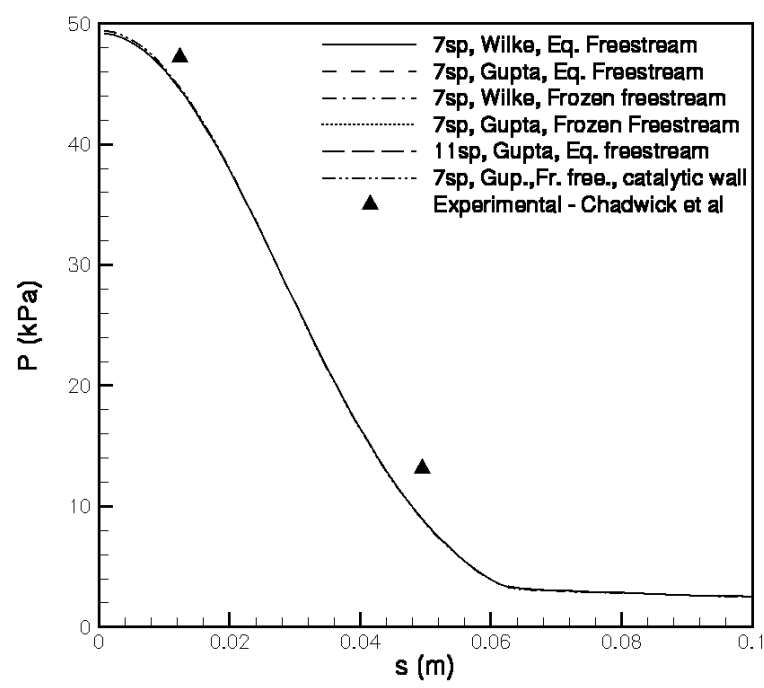

(b) Pressure

Figure 14. Dependence of results on freestream conditions and transport properties calculation method. 
wave position by a sudden increase in number density $6 \mathrm{~cm}$ away from the wall. The expansion originated at the spherical nose is visible as a decrease in electron number density occurring from 3 to $5.5 \mathrm{~cm}$ above the wall. The assumption of frozen flow changes the outer boundary of the profile but it does not change the maximum value significantly, in accordance to other researchers observations. ${ }^{48}$ The use of a 11 species chemistry model increased the electron peak density but the calculated values still do not agree well with the experimental data.

Figure 15(b) shows similar comparisons for the ES-2 position. For this case, the agreement between calculated and experimental values is further reduced. One can observe that the calculated electron densities are smaller than the values at the ES-1 position. On the other hand, the experimental electron density values are approximately the same as in the ES-1 position. The expected physical behavior of the electron density is that it should be smaller downstream because the body wall is cold and the flow is expanding along the conical section. Again, the poor comparison may indicate problems with the measurements.

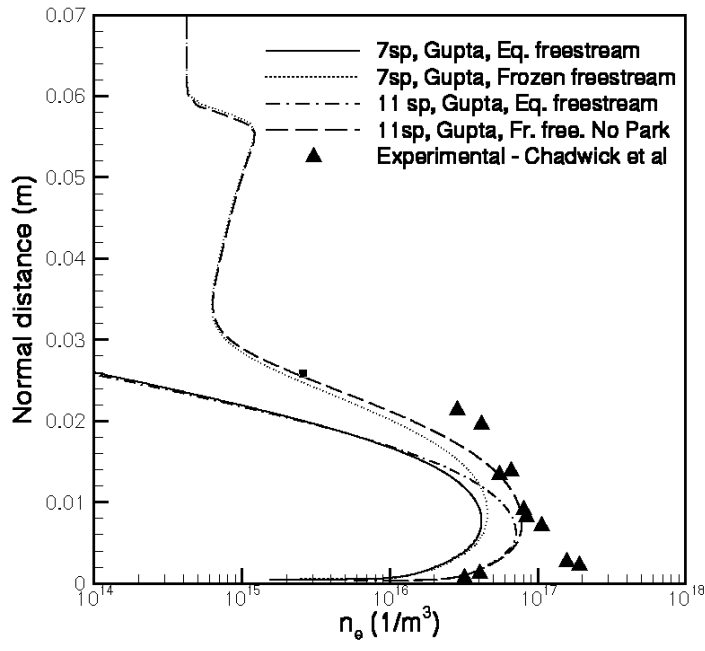

(a) ES-1 (0.198m from nose)

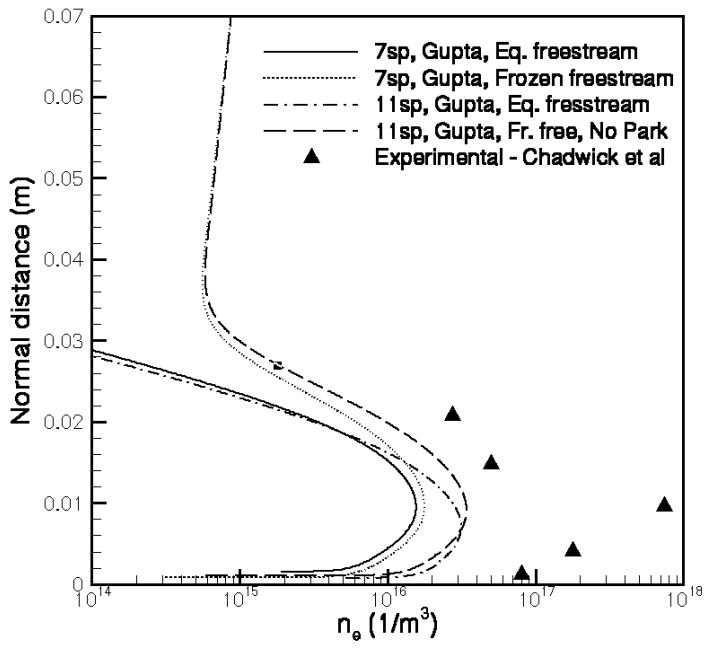

(b) ES-2 (0.47m from nose)

Figure 15. Electron number density along normal to the body.

\section{Concluding Remarks}

A code for the simulation of flows around reentry configurations is developed. The code models the flow using the Navier-Stokes equations modified to account for thermochemical nonequilibrium and weak ionization effects. The equations are solved using a finite-volume method on unstructured grids with inviscid fluxes discretized using Flux Vector Splitting. The time march is performed implicitly using a point or line implicit method. The code is also parallelized using MPI to take advantage of parallel processing.

A generalization of variable reconstruction developed for structured grids is proposed for unstructured grids. The generalization has the advantage of being readily applied to any type of elements in 2D or 3D. An important characteristic of the procedure proposed is that no interpolated values are used making the procedure very robust for hypersonic flows. Moreover, the stencil used in the reconstruction is fixed so that its cost is negligible in relation to the total cost of the calculation. The line implicit implementation used in 
the code is also demonstrated. The use of two different numbering systems can simplify the originally sparse system of equations to a tridiagonal system.

The code is successfully used to simulate the heat transfer over a Mars experimental model tested in the HYPULSE Expansion Tube. The necessity of a higher order discretization is demonstrated to obtain accurate values of heat transfer close to the symmetry axis. Numerical simulation of the RAM-C II flight experiment has also shown that the present code predictions for electron number densities agree very well with the measured data and with other researchers calculations that used the same physical models. Finally, an attempt to simulate a blunted cone experimental model in the CALSPAN 96" shock tunnel suggests that the experimental values used in the comparisons may have some problems. The measured values of heat transfer are much above calculated physical limits and the measured electron number densities do not decrease as the flow expands and cools.

\section{Acknowledgments}

The authors are indebted to the Francois-Xavier Bagnoud Foundation which provides a fellowship to the first author. Additional funding is provided by the Space Vehicle Technology Institute, under NASA grant NCC3-989 with joint sponsorship from the Department of Defense.

\section{References}

\footnotetext{
${ }^{1}$ Candler, G. V., The Computational of Weakly Ionized Flow in Nonequilibrium, Ph.D. thesis, Stanford University, California, June 1988.

${ }^{2}$ Olynick, D. R., Henline, W. D., Chambers, L. H., and Candler, G. V., "Comparison of Coupled Radiative Flow Solutions with Project Fire II Flight Data," Journal of Thermophysics and Heat Transfer, Vol. 9, 1995, pp. 586-594.

${ }^{3}$ Wright, M., Loomis, M., and Papadopoulos, P., "Aerothermal Analysis of the Project Fire II Afterbody Flow," Journal of Thermophysics and Heat Transfer, Vol. 17, 2003, pp. 240-249.

${ }^{4}$ Wright, M., Prabhu, D., and Martinez, E., "Analysis of Afterbody Heating Rates on the Apollo Command Module, Part 1: AS-2-2," AIAA Paper 2004-2456, 2004.

${ }^{5}$ Hollis, B. R., Striepe, S. A., Wright, M. J., Bose, D., Sutton, K., and Takashima, N., "Prediction of the Aerothermodynamic Environment of the Huygens Probe," AIAA Paper 2005-4816, 2005.

${ }^{6}$ Edquist, K. T., "Afterbody Heating Predictions for a Mars Science Laboratory Entry Vehicle," AIAA Paper 2005-4817, 2005.

${ }^{7}$ Gnoffo, P. A., Weilmuenster, K. J., Hamilton II, H. H., Olynick, D. R., and Venkatapathy, E., "Computational Aerothermodynamic Design Issues for Hypersonic Vehicles," Journal of Spacecraft and Rockets, Vol. 36, 1999, pp. 21-43.

${ }^{8}$ Scalabrin, L. C. and Boyd, I. D., "Development of an Unstructured Navier-Stokes Solver for Hypersonic Nonquilibrium Aerothermodynamics," AIAA Paper 2005-5203, 2005.

${ }^{9}$ Lee, J. H., "Basic Governing Equations for the Flight Regimes of Aeroassisted Orbital Transfer Vehicles," Thermal Design of Aeroassisted Orbital Transfer Vehicles, Vol. 96, AIAA, New York, 1985, pp. 3-53.

${ }^{10}$ Gnoffo, P. A., Gupta, R. N., and Shinn, J. L., "Conservation Equations and Physical Models for Hypersonic Air Flows in Thermal and Chemical Nonequilibrium," NASA-TP-2867, NASA Langley, Hampton, Virginia, 1989.

${ }^{11}$ Park, C., Nonequilibrium Hypersonic Aerothermodynamics, John Wiley \& Sons, 1990.

${ }^{12}$ Wilke, C. R., "A Viscosity Equation for Gas Mixtures," J. of Chem. Phys., Vol. 18, 1950, pp. 517-519.

${ }^{13}$ Blottner, F. G., Johnson, M., and Ellis, M., "Chemically Reacting Viscous Flow Program for Multi-Component Gas Mixtures," Sc-rr-70-754, Sandia Laboratories, Albuquerque, New Mexico, 1971.

${ }^{14}$ Vincenti, W. G. and Kruger, C. H., Introduction to Physical Gas Dynamics, Krieger Publishing Company, 1982.

${ }^{15}$ Gupta, R. N., Yos, J. M., Thompson, R. A., and Lee, K. P., "A review of reaction rates and thermodynamic and transport properties for an 11-species air model for chemical and thermal nonequilibrium calculations to 30000 K," NASA-RP-1232, 1990.

${ }^{16}$ Wright, M. J., Bose, D., Palmer, G. E., and Levin, E., "Recommended Collision Integrals for Transport Property Computations, Part 1: Air Species," AIAA Journal, Vol. 43, No. 12, 2005, pp. 2558-2564.

${ }^{17}$ Sutton, K. and Gnoffo, P. A., "Multi-Component Diffusion with Application to Computational Aerothermodynamics," AIAA Paper 1998-2575, 1998.

${ }^{18}$ Park, C., "Review of Chemical-Kinetic Problems of Future NASA Missions, I: Earth Entries," Journal of Thermophysics and Heat Transfer, Vol. 7, 1993, pp. 385-398.

${ }^{19}$ Park, C., "On Convergence of Computation of Chemically Reacting Flows," AIAA Paper 85-0247, 1985.
}

16 of 18

American Institute of Aeronautics and Astronautics 
${ }^{20}$ Millikan, R. C. and White, D. R., "Systematics of Vibrational Relaxation," J. of Chem. Phys., Vol. 39, 1963, pp. 32093213.

${ }^{21}$ Park, C., "Assessment of two-temperature kinetic model for ionizing air," AIAA Paper 1987-1574, 1987.

${ }^{22}$ Hirsch, C., Numerical Computation of Internal and External Flows, John Wiley \& Sons, 1991.

${ }^{23}$ Nompelis, I., Computational Study of Hypersonic Double-Cone Experiments for Code Validation, Ph.D. thesis, University of Minnesota, Minnesota, May 2004.

${ }^{24}$ MacCormack, R. W. and Candler, G. V., "The Solution of the Navier-Stokes Equations Using Gauss-Seidel Line Relaxation," Computers and Fluids, Vol. 17, 1989, pp. 135-150.

${ }^{25}$ Steger, J. L. and Warming, R. F., "Flux Vector Splitting for the Inviscid Gasdynamic Equations with Applications to Finite Difference Methods," Journal of Computational Physics., Vol. 40, 1981, pp. 263-293.

${ }^{26}$ Buning, P. G. and Steger, J. L., "Solution of the Two-Dimensional Euler Equations with Generalized Coordinate Transformations Using Flux Vector Splitting," AIAA Paper 82-0971, 1982.

${ }^{27}$ Druguet, M., Candler, G. V., and Nompelis, I., "Effect of Numerics on Navier-Stokes Computations of Hypersonic Double-Cone Flows," AIAA Journal, Vol. 43, No. 3, 2005, pp. 616-623.

${ }^{28}$ Jawahar, P. and Kamath, H., "A High-Resolution Procedure for Euler and Navier-Stokes Computations on Unstructured Grids," Journal of Computational Physics, Vol. 164, 2000, pp. 165-203.

${ }^{29}$ Wright, M. J., A Family of Data-Parallel Relaxation Methods for the Navier-Stokes equations, Ph.D. thesis, University of Minnesota, Minnesota, June 1997.

${ }^{30}$ Gnoffo, P. A., "Computational Aerothermodynamics in Aeroassist Applications," Journal of Spacecrafts and Rockets, Vol. 40, No. 3, 2003, pp. 305-312.

${ }^{31}$ Venkatakrishnan, V., "Implicit Schemes and Parallel Computing in Unstructured Grid CFD," VKI-LS-1995-02, VKI Lecture Series, 1995.

${ }^{32}$ Barth, T. J. and Jespersen, D. C., "The Design and Application of Upwind Schemes on Unstructured Meshes," AIAA Paper 1989-0366, 1989.

${ }^{33}$ Kim, S., Makarov, B., and Caraeni, D., "A Multi-Dimensional Linear Reconstruction Scheme for Arbitrary Unstructured Grids," AIAA Paper 2003-3990, 2003.

${ }^{34}$ Venkatakrishnan, V., "Convergence to Steady State Solutions of the Euler Equations on Unstructured Grids with Limiters," Journal of Computational Physics, Vol. 118, 1995, pp. 120-130.

${ }^{35}$ Gnoffo, P. A., "Computational Aerothermodynamic Simulation Issues on Unstructured Grids," AIAA Paper 2004-2371, 2004.

${ }^{36}$ Nompelis, I., Drayna, T. W., and Candler, G. V., "A Parallel Unstructured Implicit Solver for Reacting Flow Simulation," AIAA Paper 2005-4867, 2005.

${ }^{37}$ Batina, J., "Three-Dimensional Flux-Split Euler Schemes Involving Unstructured Dynamic Meshes," AIAA Paper 1990$1649,1990$.

${ }^{38}$ Bibb, K. L., Peraire, J., and Riley, C. J., "Hypersonic Flow Computations on Unstructured Meshes," AIAA Paper 1997-0625, 1997

${ }^{39}$ Harten, A., Engquist, B., Osher, S., and Chakravarthy, S., "Uniformly high order essentially non-oscillatory schemes, III," Journal of Computational Physics, Vol. 71, 1987, pp. 231-303.

${ }^{40} \mathrm{Shu}, \mathrm{C} . \mathrm{W}$. and Osher, S., "Efficient implementation of essentially non-oscilatory shock-capturing schemes," Journal of Computational Physics, Vol. 77, No. 2, 1988, pp. 439-471.

${ }^{41}$ Liu, X. D., Osher, S., and Chan, T., "Weighted essentially nonoscillatory schemes," Journal of Computational Physics, Vol. 115, 1994, pp. 200-212.

${ }^{42}$ Karypis, G. and Kumar, V., METIS: A Software Package for Partitioning Unstructured Graphs, Partitioning Meshes, and Computing Fill-Reducing Orderings of Sparse Matrices, Univeristy of Minnesota, MN, 1998.

${ }^{43}$ Hollis, B. R. and Perkins, J. N., "High-Enthalpy Aerothermodynamics of a Mars Entry Vehicle Part 1: Experimental Results," Journal of Spacecraft and Rockets, Vol. 34, No. 4, 1997, pp. 449-456.

${ }^{44}$ Hollis, B. R. and Perkins, J. N., "High-Enthalpy Aerothermodynamics of a Mars Entry Vehicle Part 2: Computational Results," Journal of Spacecraft and Rockets, Vol. 34, No. 4, 1997, pp. 457-463.

${ }^{45}$ Hollis, B. R., "Experimental and Computational Aerothermodynamics of a Mars Entry Vehicle," NASA-CR-201633, Dec. 1996.

${ }^{46}$ Chadwick, K. M., Boyer, D. W., and Andre, S. S., "Plasma and Flowfield Induced Effects on Hypervelocity Re-entry Vehicles for L-Band Irradiation at Near Broadside Aspect Angles," AIAA Paper 1996-2322, 1996.

${ }^{47}$ Chadwick, K. M., Boyer, D. W., and Andre, S. S., "Plasma and Flowfield Induced Effects on Re-entry Vehicles for L-Band at Near Broadside Aspect Angles," PL-TR-2094, U.S. Air Force Philips Lab., 1996.

${ }^{48}$ Lordi, J. A., Boyer, D. W., Dunn, M. G., Smolarek, K. K., and Wittliff, C. E., "Description of Nonequilibrium Effects on Simulation of Flows about Hypersonic Vehicles," AIAA Paper 1988-0476, 1988.

${ }^{49}$ Grasso, F. and Capano, G., "Modeling of Ionizing Hypersonic Flows in Nonequilibrium," Journal of Spacecraft and Rockets, Vol. 32, No. 2, 1995, pp. 217-224.

${ }^{50}$ Josyula, E. and Bailey, W., "Governing Equations for Weakly Ionized Plasma Flowfields of Aerospace Vehicles," Journal of Spacecraft and Rockets, Vol. 40, No. 6, 2003, pp. 845-857.

17 of 18

American Institute of Aeronautics and Astronautics 
${ }^{51}$ Victoria, K. J. and Widhopf, G. F., "Numerical Solution of the Unsteady Navier-Stokes Equations in Curvilinear Coordinates: The Hypersonic Blunt Body Merged Layer Problem," Proceedings of the 3rd International Conference on Numerical Methods in Fluid Dynamics, Paris, France, 1972, pp. 179-188.

${ }^{52}$ Palmer, G. and Wright, M., "Comparisons of Methods to Compute High-Temperature Gas Viscosity," Journal of Thermophysics and Heat Transfer, Vol. 17, No. 2, 2003, pp. 232-239.

${ }^{53}$ Nompelis, I., Candler, G. V., Holden, M. S., and Wadhams, T. P., "Real Gas Effects on Hypersonic Shock Wave Laminar Boundary Interactions," AIAA Paper 2003-0443, 2003.

${ }^{54}$ Park, C., "Chemical-Kinetic Parameters of Hyperbolic Earth Entry," Journal of Thermophysics and Heat Transfer, Vol. 15, 2001, pp. 76-90. 